ORIGINAL ARTICLE

\section{Dapagliflozin in Patients with Chronic Kidney Disease}

\author{
Hiddo J.L. Heerspink, Ph.D., Bergur V. Stefánsson, M.D., \\ Ricardo Correa-Rotter, M.D., Glenn M. Chertow, M.D., Tom Greene, Ph.D., \\ Fan-Fan Hou, M.D., Johannes F.E. Mann, M.D., John J.V. McMurray, M.D., \\ Magnus Lindberg, M.Sc., Peter Rossing, M.D., C. David Sjöström, M.D., \\ Roberto D. Toto, M.D., Anna-Maria Langkilde, M.D., and David C. Wheeler, M.D., \\ for the DAPA-CKD Trial Committees and Investigators*
}

ABSTRACT

BACKGROUND

Patients with chronic kidney disease have a high risk of adverse kidney and cardiovascular outcomes. The effect of dapagliflozin in patients with chronic kidney disease, with or without type 2 diabetes, is not known.

\section{METHODS}

We randomly assigned 4304 participants with an estimated glomerular filtration rate (GFR) of 25 to $75 \mathrm{ml}$ per minute per $1.73 \mathrm{~m}^{2}$ of body-surface area and a urinary albumin-to-creatinine ratio (with albumin measured in milligrams and creatinine measured in grams) of 200 to 5000 to receive dapagliflozin (10 mg once daily) or placebo. The primary outcome was a composite of a sustained decline in the estimated GFR of at least $50 \%$, end-stage kidney disease, or death from renal or cardiovascular causes.

\section{RESULTS}

The independent data monitoring committee recommended stopping the trial because of efficacy. Over a median of 2.4 years, a primary outcome event occurred in 197 of 2152 participants (9.2\%) in the dapagliflozin group and 312 of 2152 participants $(14.5 \%)$ in the placebo group (hazard ratio, $0.61 ; 95 \%$ confidence interval [CI], 0.51 to $0.72 ; \mathrm{P}<0.001$; number needed to treat to prevent one primary outcome event, 19 [95\% CI, 15 to 27]). The hazard ratio for the composite of a sustained decline in the estimated GFR of at least $50 \%$, end-stage kidney disease, or death from renal causes was $0.56(95 \% \mathrm{CI}, 0.45$ to $0.68 ; \mathrm{P}<0.001)$, and the hazard ratio for the composite of death from cardiovascular causes or hospitalization for heart failure was 0.71 ( $95 \%$ CI, 0.55 to $0.92 ; \mathrm{P}=0.009)$. Death occurred in 101 participants $(4.7 \%)$ in the dapagliflozin group and 146 participants $(6.8 \%)$ in the placebo group (hazard ratio, $0.69 ; 95 \% \mathrm{CI}, 0.53$ to $0.88 ; \mathrm{P}=0.004$ ). The effects of dapagliflozin were similar in participants with type 2 diabetes and in those without type 2 diabetes. The known safety profile of dapagliflozin was confirmed.

CONCLUSIONS

Among patients with chronic kidney disease, regardless of the presence or absence of diabetes, the risk of a composite of a sustained decline in the estimated GFR of at least $50 \%$, end-stage kidney disease, or death from renal or cardiovascular causes was significantly lower with dapagliflozin than with placebo. (Funded by AstraZeneca; DAPA-CKD ClinicalTrials.gov number, NCT03036150.)
The authors' affiliations are listed in the Appendix. Address reprint requests to Dr. Heerspink at the Department of Clinical Pharmacy and Pharmacology, University of Groningen, P.O. Box 30.001, 9700 RB Groningen, the Netherlands, or at h.j.lambers.heerspink@umcg.nl.

*A complete list of DAPA-CKD committee members and investigators is provided in the Supplementary Appendix, available at NEJM.org.

This article was published on September 24, 2020, at NEJM.org.

DOI: 10.1056/NEJMoa2024816 Copyright (c) 2020 Massachusetts Medical Society. 
W

ORLDWIDE ESTIMATES INDICATE that nearly 700 million persons have chronic kidney disease. ${ }^{1}$ Chronic kidney disease is an important contributor to illness and is associated with a diminished quality of life and a reduced life expectancy. Despite the widespread availability of simple laboratory tests to identify patients with impaired kidney function, fewer clinical trials have been conducted for kidney diseases than for other common medical conditions. ${ }^{2}$ Until recently, the only classes of medication that have been shown to slow a decline in kidney function were angiotensin-convertingenzyme (ACE) inhibitors and angiotensin-receptor blockers (ARBs); however, most of the evidence was generated in patients with type 2 diabetes. ${ }^{3-6}$

Sodium-glucose cotransporter 2 (SGLT2) inhibitors decrease glycated hemoglobin levels and have shown favorable effects on kidney and cardiovascular outcomes in large clinical trials involving patients with type 2 diabetes.-9 The CREDENCE (Canagliflozin and Renal Events in Diabetes with Established Nephropathy Clinical Evaluation) trial showed that long-term administration of canagliflozin conferred renal and cardiovascular protection in patients with type 2 diabetes with chronic kidney disease. ${ }^{10}$ Although the underlying mechanisms are not completely understood, the benefits of SGLT2 inhibitors appear to be independent of their blood glucoselowering effects and may be mediated by natriuresis and glucose-induced osmotic diuresis, leading to a reduction in intraglomerular pressure. ${ }^{11-13}$ This favorable hemodynamic effect may also preserve kidney function in persons with kidney diseases due to causes other than type 2 diabetes. We designed the Dapagliflozin and Prevention of Adverse Outcomes in Chronic Kidney Disease (DAPA-CKD) trial to assess the longterm efficacy and safety of the SGLT2 inhibitor dapagliflozin in patients with chronic kidney disease, with or without type 2 diabetes.

\section{METHODS}

TRIAL DESIGN AND OVERSIGHT

Our trial was a randomized, double-blind, placebo-controlled, multicenter clinical trial; details regarding the trial design and baseline characteristics of the participants have been published previously. ${ }^{14,15}$ The trial was sponsored by AstraZeneca and conducted at 386 sites in 21 coun- tries from February 2, 2017, through June 12, 2020. An executive committee consisting of nine academic members and two nonvoting employees of the sponsor was responsible for the design and oversight of the trial and the reporting of the results. The trial protocol (available with the full text of this article at NEJM.org) was approved by a central or local ethics committee at each trial site. All the participants provided written informed consent before any trial-specific procedure commenced. The safety of the participants was overseen by an independent data monitoring committee. The analyses that were conducted by the sponsor were replicated by an independent academic group at the University Medical Center Groningen. The first draft of the manuscript was written by the first and last authors and was revised by the coauthors. The decision to submit the manuscript for publication was made jointly by all the authors. The first and last authors vouch for the completeness and accuracy of the data and for the fidelity of the trial to the protocol.

\section{PARTICIPANTS}

Adults with or without type 2 diabetes who had an estimated glomerular filtration rate (GFR) of 25 to $75 \mathrm{ml}$ per minute per $1.73 \mathrm{~m}^{2}$ of body-surface area and a urinary albumin-to-creatinine ratio (with albumin measured in milligrams and creatinine measured in grams) of 200 to 5000 were eligible for participation. All the participants were required to be receiving a stable dose of an ACE inhibitor or ARB for at least 4 weeks before screening. However, participants who were documented to be unable to take ACE inhibitors or ARBs were allowed to participate. Key exclusion criteria were a documented diagnosis of type 1 diabetes, polycystic kidney disease, lupus nephritis, or antineutrophil cytoplasmic antibodyassociated vasculitis. Participants who had received immunotherapy for primary or secondary kidney disease within 6 months before enrollment were also excluded. The full inclusion and exclusion criteria are listed in the Supplementary Appendix, available at NEJM.org.

\section{TRIAL PROCEDURES}

Participants were randomly assigned to receive dapagliflozin (10 mg once daily) or matching placebo, in accordance with the sequestered, fixedrandomization schedule, with the use of balanced 
blocks to ensure an approximate 1:1 ratio of the two regimens. Randomization was stratified according to the diagnosis of type 2 diabetes (yes or no) and the urinary albumin-to-creatinine ratio ( $\leq 1000$ or $>1000$ ). Investigators used an interactive voice-response or Web-response system to determine trial-group assignments. Randomization was monitored to ensure that a minimum of $30 \%$ of the participants were recruited to either the population with type 2 diabetes or the population without diabetes. Recruitment of participants with an estimated GFR of 60 to $75 \mathrm{ml}$ per minute per $1.73 \mathrm{~m}^{2}$ was halted on November 27, 2017 , to ensure that no more than $10 \%$ of the trial participants had stage 2 chronic kidney disease. Participants and all trial personnel (except the members of the independent data monitoring committee) were unaware of the trial-group assignments. Dapagliflozin and placebo were packaged identically, with uniform tablet appearance, labeling, and administration schedules.

After randomization, in-person trial visits were performed at 2 weeks, at 2, 4, and 8 months, and at 4-month intervals thereafter (Fig. S1 in the Supplementary Appendix). At each follow-up visit, vital signs were recorded, blood and urine samples were sent for laboratory assessment, and information on potential trial outcomes, adverse events, concomitant therapies, and adherence to the trial regimen was collected. Dapagliflozin or placebo was to be discontinued if pregnancy or diabetic ketoacidosis occurred. Before trial completion, each participant underwent a final trial visit.

\section{OUTCOMES}

The primary composite outcome, assessed in a time-to-event analysis, was the first occurrence of any of the following: a decline of at least $50 \%$ in the estimated GFR (confirmed by a second serum creatinine measurement after $\geq 28$ days), the onset of end-stage kidney disease (defined as maintenance dialysis for $\geq 28$ days, kidney transplantation, or an estimated GFR of $<15 \mathrm{ml}$ per minute per $1.73 \mathrm{~m}^{2}$ confirmed by a second measurement after $\geq 28$ days), or death from renal or cardiovascular causes. Secondary outcomes (also assessed in time-to-event analyses) were, in hierarchical order, the composite kidney outcome of a sustained decline in the estimated GFR of at least $50 \%$, end-stage kidney disease, or death from renal causes; a composite cardiovascular outcome defined as hospitalization for heart failure or death from cardiovascular causes; and death from any cause. An independent committee whose members were unaware of the trial-group assignments adjudicated all primary and secondary outcomes, except for a sustained decline in the estimated GFR of at least $50 \%$ and a sustained estimated GFR of less than $15 \mathrm{ml}$ per minute per $1.73 \mathrm{~m}^{2}$, which were ascertained from central laboratory measurements. Members of the eventadjudication committee and the outcome definitions are reported in the Supplementary Appendix.

Selected adverse-event data were collected in the trial. These included serious adverse events, adverse events resulting in the discontinuation of dapagliflozin or placebo, and adverse events of interest (symptoms of volume depletion, renal events, major hypoglycemia, bone fractures, amputations, and potential diabetic ketoacidosis). Potential cases of diabetic ketoacidosis were adjudicated by the independent adjudication committee. All events suggestive of Fournier's gangrene were evaluated by an internal safety group consisting of representatives of the sponsor who were unaware of the trial-group assignments.

\section{STATISTICAL ANALYSIS}

The analytical approach and power calculations have been published previously, ${ }^{14}$ and the complete prespecified statistical analysis plan is available with the protocol at NEJM.org. In brief, a formal interim analysis was originally planned when $75 \%$ of primary outcome events had occurred. However, during the conduct of the trial it became apparent that the interim analysis would occur close to the end of the trial. The executive committee therefore decided to remove the interim analysis from the protocol. We estimated that 681 primary outcome events would be needed to detect a $22 \%$ lower relative risk in the dapagliflozin group than in the placebo group (hazard ratio of 0.78 ), with $90 \%$ power using a two-sided alpha level of 0.05 and assuming an annual event rate for the primary outcome of $7.5 \%$ in the placebo group. A closed testing procedure that included a prespecified hierarchical order of the primary and secondary outcomes was used to ensure control of type I error at a two-sided 0.05 level.

The primary efficacy analysis was based on the intention-to-treat population, which included all the participants who had undergone random- 
ization. A Cox proportional-hazards regression model that was stratified according to the factors used at randomization (type 2 diabetes and urinary albumin-to-creatinine ratio) and that was adjusted for the baseline estimated GFR was used to estimate the hazard ratio and $95 \%$ confidence intervals for dapagliflozin as compared with placebo for the primary and secondary outcomes. Data were censored on April 3, 2020, or the date of the last central laboratory assessment, clinical assessment, or known contact, depending on the specific outcome. Prespecified subgroups were assessed with the use of the same stratified Cox proportional-hazards model without adjustment for multiple comparisons. A mixed model for repeated measurements was used to analyze changes in the estimated GFR in the on-treatment analysis population. The model was adjusted for the baseline value, trial-group assignment, visit, and the interaction between trial-group assignment and visit. All available on-treatment measurements were used with no distinction made for missing outcomes for participants who were alive and outcomes that were not observed because of death. The effect of dapagliflozin as compared with placebo on the rate of decline in the estimated GFR during the acute phase (baseline to week 2), chronic phase (week 2 until end of treatment), and total slope to month 30 was analyzed with the use of a twoslope model; details are provided in the Supplementary Appendix.

Safety data are summarized according to trial group with the use of the data set for all the participants who had undergone randomization and received at least one dose of dapagliflozin or placebo. Safety analyses were performed on all adverse events occurring before or at the trial closure visit. All analyses were performed with SAS software, version 9.4 (SAS Institute).

\section{RESULTS}

\section{PARTICIPANTS AND FOLLOW-UP}

From February 2017 through October 2018, a total of 7517 participants were screened, of whom 4094 underwent randomization. Because of regulatory delays, enrollment in China did not commence until December 2019, after which 210 participants from China underwent randomization until March 2020. Details about the randomization and follow-up of the participants are provided in Figure S2. The baseline characteristics, including medications for type 2 diabetes and kidney disease, were balanced between the dapagliflozin and placebo groups (Table 1). The mean $( \pm S D)$ age was $61.8 \pm 12.1$ years, and 1425 participants (33.1\%) were female. The mean estimated GFR was $43.1 \pm 12.4 \mathrm{ml}$ per minute per $1.73 \mathrm{~m}^{2}$, the median urinary albumin-to-creatinine ratio was 949, and 2906 participants (67.5\%) had received a diagnosis of type 2 diabetes.

After a regular review meeting on March 26, 2020, the independent data monitoring committee recommended to the two coprincipal investigators (the first and last authors) that the trial be discontinued because of clear efficacy, on the basis of 408 primary outcome events. The trial leadership team accepted this recommendation and chose April 3, 2020, as the cutoff date for all efficacy analyses.

At the conclusion of the trial, the median follow-up was 2.4 years (interquartile range, 2.0 to 2.7). Dapagliflozin was discontinued for reasons other than death in 274 participants, and placebo was discontinued in 309 participants $(12.7 \%$ and $14.4 \%$, respectively). In all, 4289 participants $(99.7 \%)$ completed the trial (i.e., were alive with follow-up data available at the trial completion visit or had died during follow-up). A total of 11 participants $(0.3 \%)$ withdrew consent, and vital status was ascertained for all but 5 participants (0.1\%).

\section{EFFICACY OUTCOMES}

The primary composite outcome of a sustained decline in the estimated GFR of at least 50\%, end-stage kidney disease, or death from renal or cardiovascular causes occurred in 197 participants $(9.2 \%)$ in the dapagliflozin group and 312 participants (14.5\%) in the placebo group (hazard ratio, $0.61 ; 95 \%$ confidence interval [CI], 0.51 to 0.72; $\mathrm{P}<0.001$ ) (Table 2 and Fig. $1 \mathrm{~A}$ ). The event rates for all components of the composite outcome favored dapagliflozin (Table 2). The number of participants who needed to be treated during the trial period to prevent one primary outcome event was 19 (95\% CI, 15 to 27).

The effect of dapagliflozin on the primary outcome was generally consistent across prespecified subgroups (Fig. 2). In participants with type 2 diabetes, the hazard ratio for the comparison of dapagliflozin and placebo for the primary outcome was 0.64 ( $95 \%$ CI, 0.52 to 0.79 ), as com- 


\begin{tabular}{|c|c|c|}
\hline Characteristic & $\begin{array}{l}\text { Dapagliflozin } \\
\text { (N=2152) }\end{array}$ & $\begin{array}{l}\text { Placebo } \\
(\mathrm{N}=2152)\end{array}$ \\
\hline Age $-y r$ & $61.8 \pm 12.1$ & $61.9 \pm 12.1$ \\
\hline Female sex — no. (\%) & 709 (32.9) & $716(33.3)$ \\
\hline \multicolumn{3}{|l|}{ Race - no. $(\%) \dagger$} \\
\hline White & $1124(52.2)$ & $1166(54.2)$ \\
\hline Black & $104(4.8)$ & $87(4.0)$ \\
\hline Asian & $749(34.8)$ & $718(33.4)$ \\
\hline Other & $175(8.1)$ & $181(8.4)$ \\
\hline Weight — kg & $81.5 \pm 20.1$ & $82.0 \pm 20.9$ \\
\hline Body-mass indext & $29.4 \pm 6.0$ & $29.6 \pm 6.3$ \\
\hline Current smoker — no. (\%) & $283(13.2)$ & $301(14.0)$ \\
\hline \multicolumn{3}{|l|}{ Blood pressure - $\mathrm{mm} \mathrm{Hg}$} \\
\hline Systolic & $136.7 \pm 17.5$ & $137.4 \pm 17.3$ \\
\hline Diastolic & $77.5 \pm 10.7$ & $77.5 \pm 10.3$ \\
\hline \multicolumn{3}{|l|}{ Estimated GFR } \\
\hline Mean $-\mathrm{ml} / \mathrm{min} / 1.73 \mathrm{~m}^{2}$ & $43.2 \pm 12.3$ & $43.0 \pm 12.4$ \\
\hline \multicolumn{3}{|l|}{ Distribution — no. (\%) } \\
\hline$\geq 60 \mathrm{ml} / \mathrm{min} / 1.73 \mathrm{~m}^{2}$ & $234(10.9)$ & $220(10.2)$ \\
\hline 45 to $<60 \mathrm{ml} / \mathrm{min} / 1.73 \mathrm{~m}^{2}$ & $646(30.0)$ & $682(31.7)$ \\
\hline 30 to $<45 \mathrm{ml} / \mathrm{min} / 1.73 \mathrm{~m}^{2}$ & $979(45.5)$ & $919(42.7)$ \\
\hline$<30 \mathrm{ml} / \mathrm{min} / 1.73 \mathrm{~m}^{2}$ & $293(13.6)$ & $331(15.4)$ \\
\hline Hemoglobin — g/liter & $128.6 \pm 18.1$ & $127.9 \pm 18.0$ \\
\hline Serum potassium $-\mathrm{mEq} /$ liter & $4.6 \pm 0.5$ & $4.6 \pm 0.6$ \\
\hline \multicolumn{3}{|l|}{ Urinary albumin-to-creatinine ratiof } \\
\hline Median (interquartile range) & $965(472-1903)$ & $934(482-1868)$ \\
\hline$>1000-$ no. (\%) & $1048(48.7)$ & $1031(47.9)$ \\
\hline Type 2 diabetes — no. (\%) & $1455(67.6)$ & $1451(67.4)$ \\
\hline Cardiovascular disease - no. (\%) & $813(37.8)$ & $797(37.0)$ \\
\hline Heart failure - no. (\%) & $235(10.9)$ & $233(10.8)$ \\
\hline \multicolumn{3}{|l|}{ Previous medication — no. (\%) } \\
\hline ACE inhibitor & $673(31.3)$ & $681(31.6)$ \\
\hline ARB & $1444(67.1)$ & $1426(66.3)$ \\
\hline Diuretic & $928(43.1)$ & $954(44.3)$ \\
\hline Statin & $1395(64.8)$ & $1399(65.0)$ \\
\hline
\end{tabular}

* Plus-minus values are mean \pm SD. Percentages may not total 100 because of rounding. ACE denotes angiotensin-converting enzyme, ARB angiotensin-receptor blocker, and GFR glomerular filtration rate.

$\dagger$ Race was reported by the investigators; the designation "other" includes Native Hawaiian or other Pacific Islander, American Indian or Alaska Native, and other.

$t$ The body-mass index is the weight in kilograms divided by the square of the height in meters.

$\int$ The albumin-to-creatinine ratio was calculated with albumin measured in milligrams and creatinine measured in grams.

q Cardiovascular disease was defined as a history of peripheral artery disease, angina pectoris, myocardial infarction, percutaneous coronary intervention, coronary-artery bypass grafting, heart failure, valvular heart disease, abdominal aorta aneurysm, atrial fibrillation, atrial flutter, ischemic stroke, transient ischemic attack, hemorrhagic stroke, carotid artery stenosis, cardiac-pacemaker insertion, vascular stent, coronary-artery stenosis, ventricular arrhythmia, implantable cardioverter-defibrillator, noncoronary revascularization, or surgical amputation.

The New England Journal of Medicine 


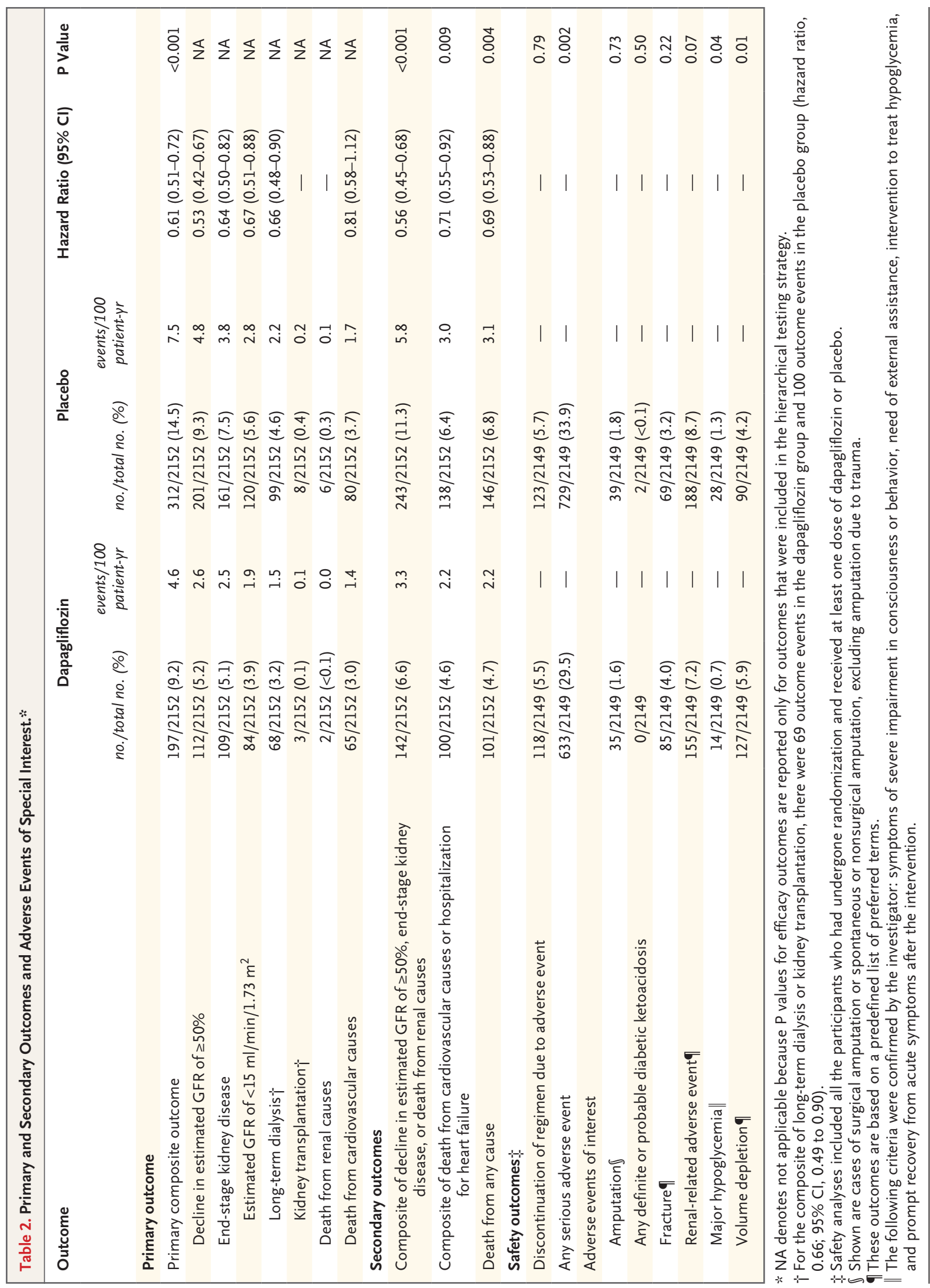




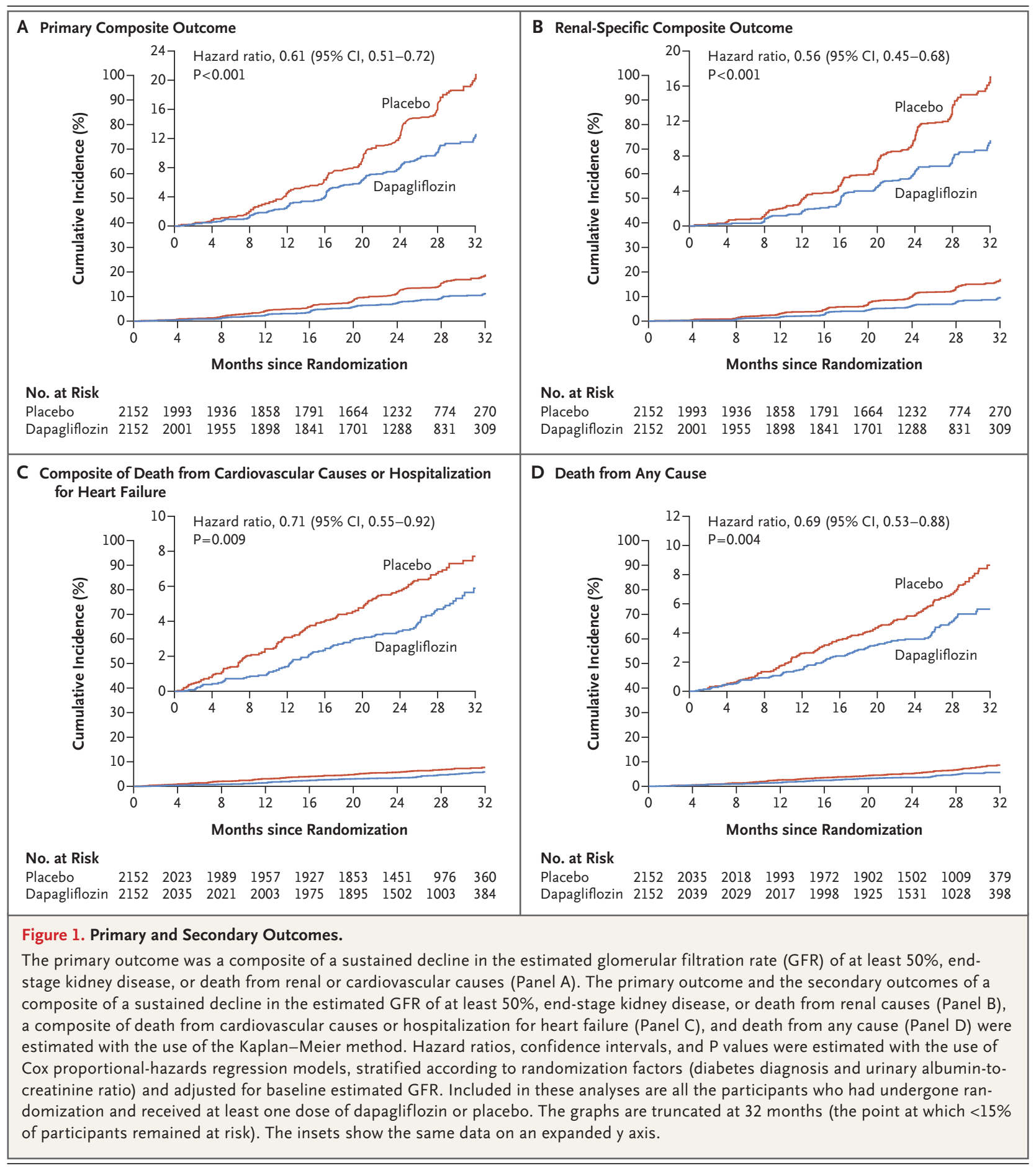

pared with 0.50 (95\% CI, 0.35 to 0.72 ) in participants without type 2 diabetes.

The incidence of each secondary outcome was lower in the dapagliflozin group than in the placebo group (Table 2). The hazard ratio for the kidney composite of a sustained decline in the estimated GFR of at least $50 \%$, end-stage kidney disease, or death from renal causes was 0.56 (95\% CI, 0.45 to 0.68 ; $\mathrm{P}<0.001$ ) (Table 2 and Fig. 1B). The hazard ratio for the composite of death from cardiovascular causes or hospitalization for heart failure was 0.71 ( $95 \%$ CI, 0.55 to 


\begin{tabular}{|c|c|c|c|c|}
\hline \multirow[t]{2}{*}{ Subgroup } & Dapagliflozin & Placebo & \multicolumn{2}{|c|}{ Hazard Ratio $(95 \% \mathrm{CI})$} \\
\hline & \multicolumn{2}{|c|}{ no. of participants/total no. } & & \\
\hline All participants & $197 / 2152$ & $312 / 2152$ & $\longmapsto$ & $0.61(0.51-0.72)$ \\
\hline \multicolumn{5}{|l|}{ Age } \\
\hline$\leq 65 \mathrm{yr}$ & $122 / 1247$ & $191 / 1239$ & $\longmapsto$ & $0.64(0.51-0.80)$ \\
\hline$>65 \mathrm{yr}$ & $75 / 905$ & $121 / 913$ & $\longmapsto$ & $0.58(0.43-0.77)$ \\
\hline \multicolumn{5}{|l|}{ Sex } \\
\hline Male & $126 / 1443$ & $209 / 1436$ & $\longmapsto$ & $0.57(0.46-0.72)$ \\
\hline Female & $71 / 709$ & $103 / 716$ & $\longmapsto$ & $0.65(0.48-0.88)$ \\
\hline \multicolumn{5}{|l|}{ Race } \\
\hline White & $110 / 1124$ & $174 / 1166$ & $\longmapsto$ & $0.62(0.49-0.79)$ \\
\hline Black & $7 / 104$ & $14 / 87$ & $\longrightarrow$ & $0.33(0.13-0.81)$ \\
\hline Asian & $53 / 749$ & $77 / 718$ & $\longmapsto$ & $0.66(0.46-0.93)$ \\
\hline Other & $27 / 175$ & $47 / 181$ & $\longmapsto$ & $0.54(0.33-0.86)$ \\
\hline \multicolumn{5}{|l|}{ Geographic region } \\
\hline Asia & $50 / 692$ & $69 / 654$ & $\longmapsto-1$ & $0.70(0.48-1.00)$ \\
\hline Europe & $57 / 610$ & $89 / 623$ & $\longmapsto$ & $0.60(0.43-0.85)$ \\
\hline North America & $35 / 401$ & $69 / 412$ & $\longmapsto$ & $0.51(0.34-0.76)$ \\
\hline Latin America & $55 / 449$ & $85 / 463$ & $\longmapsto$ & $0.61(0.43-0.86)$ \\
\hline \multicolumn{5}{|l|}{ Type 2 diabetes } \\
\hline Yes & $152 / 1455$ & $229 / 1451$ & $\longmapsto$ & $0.64(0.52-0.79)$ \\
\hline No & $45 / 697$ & $83 / 701$ & $\longmapsto$ & $0.50(0.35-0.72)$ \\
\hline \multicolumn{5}{|l|}{ Estimated GFR } \\
\hline$<45 \mathrm{ml} / \mathrm{min} / 1.73 \mathrm{~m}^{2}$ & $152 / 1272$ & $217 / 1250$ & $\longmapsto$ & $0.63(0.51-0.78)$ \\
\hline$\geq 45 \mathrm{ml} / \mathrm{min} / 1.73 \mathrm{~m}^{2}$ & $45 / 880$ & $95 / 902$ & $\longmapsto$ & $0.49(0.34-0.69)$ \\
\hline \multicolumn{5}{|c|}{ Urinary albumin-to-creatinine ratio } \\
\hline$\leq 1000$ & $44 / 1104$ & $84 / 1121$ & $\longmapsto$ & $0.54(0.37-0.77)$ \\
\hline$>1000$ & $153 / 1048$ & $228 / 1031$ & $\longmapsto$ & $0.62(0.50-0.76)$ \\
\hline \multicolumn{5}{|l|}{ Systolic blood pressure } \\
\hline$\leq 130 \mathrm{~mm} \mathrm{Hg}$ & $46 / 793$ & $96 / 749$ & $\longmapsto$ & $0.44(0.31-0.63)$ \\
\hline \multirow[t]{3}{*}{$>130 \mathrm{~mm} \mathrm{Hg}$} & $151 / 1359$ & $216 / 1403$ & $\longmapsto$ & $0.68(0.56-0.84)$ \\
\hline & & 0.1 & $0.5 \quad 1.0$ & \\
\hline & & & ozin Better & Better \\
\hline \multicolumn{5}{|c|}{ Figure 2. Primary Outcome According to Prespecified Subgroups at Baseline. } \\
\hline \multicolumn{5}{|c|}{$\begin{array}{l}\text { Shown are forest plots of the hazard ratios for the primary outcome (a composite of a sustained decline in the esti- } \\
\text { mated GFR of } \geq 50 \% \text {, end-stage kidney disease, or death from renal or cardiovascular causes) according to prespeci- } \\
\text { fied baseline subgroups. Hazard ratios and confidence intervals were calculated with a Cox proportional-hazards } \\
\text { model with stratification according to diabetes status and urinary albumin-to-creatinine ratio adjusted for baseline } \\
\text { estimated GFR, with factors for trial group, subgroup, and the interaction between trial group and the subgroup } \\
\text { variable. Race was reported by the investigators. The albumin-to-creatinine ratio was calculated with albumin mea- } \\
\text { sured in milligrams and creatinine measured in grams. }\end{array}$} \\
\hline
\end{tabular}

0.92; $\mathrm{P}=0.009$ ) (Table 2 and Fig. $1 \mathrm{C}$ ). There were 101 deaths (4.7\%) from any cause in the dapagliflozin group and $146(6.8 \%)$ in the placebo group (hazard ratio, 0.69 ; $95 \%$ CI, 0.53 to 0.88 ; $\mathrm{P}=0.004$ ) (Table 2 and Fig. 1D).

The least-squares mean $( \pm$ SE) estimated GFR slopes from baseline to 30 months in the dapagliflozin and placebo groups were $-2.86 \pm 0.11$ and $-3.79 \pm 0.11 \mathrm{ml}$ per minute per $1.73 \mathrm{~m}^{2}$ per year, respectively, resulting in a between-group difference of $0.93 \mathrm{ml}$ per minute per $1.73 \mathrm{~m}^{2}$ per year
(95\% CI, 0.61 to 1.25) (Fig. 3). During the first 2 weeks, there was a greater reduction in the estimated GFR in the dapagliflozin group than in the placebo group $(-3.97 \pm 0.15$ vs. $-0.82 \pm 0.15 \mathrm{ml}$ per minute per $\left.1.73 \mathrm{~m}^{2}\right)$. Thereafter, the annual change in the mean estimated GFR was smaller with dapagliflozin than with placebo $(-1.67 \pm 0.11$ and $-3.59 \pm 0.11 \mathrm{ml}$ per minute per $1.73 \mathrm{~m}^{2}$, respectively), for a between-group difference of $1.92 \mathrm{ml}$ per minute per $1.73 \mathrm{~m}^{2}$ per year $(95 \% \mathrm{CI}$, 1.61 to 2.24$)$. 


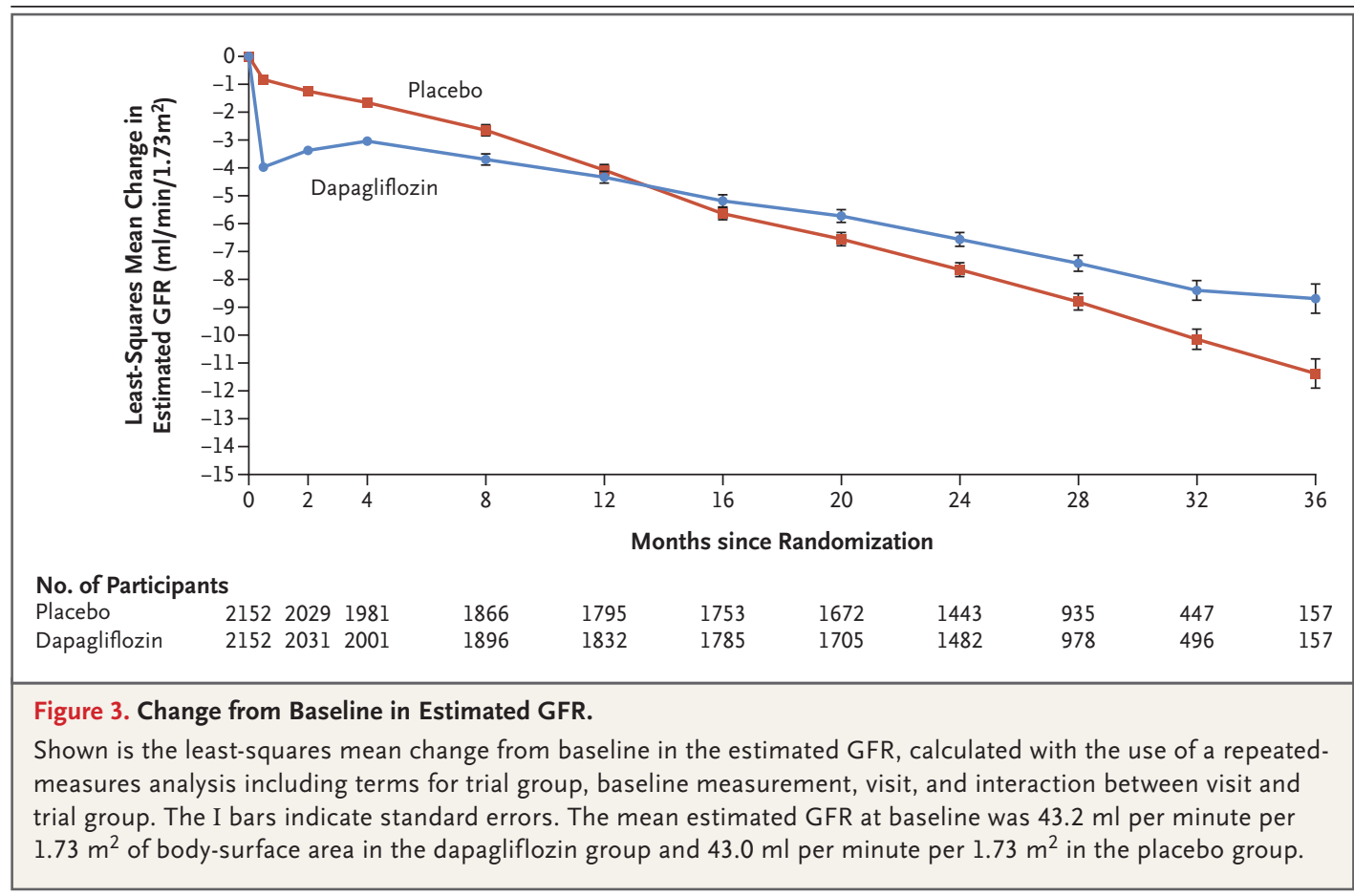

\section{SAFETY OUTCOMES AND ADVERSE EVENTS}

The incidences of adverse events and serious adverse events were similar overall in the dapagliflozin and placebo groups (Table 2). Diabetic ketoacidosis was not reported in any participants who received dapagliflozin and in two participants who received placebo. Neither diabetic ketoacidosis nor severe hypoglycemia was observed in participants without type 2 diabetes. There was one confirmed case of Fournier's gangrene in the placebo group and none in the dapagliflozin group.

\section{DISCUSSION}

We found that participants with chronic kidney disease, with or without type 2 diabetes, who were randomly assigned to receive dapagliflozin had a lower risk of the primary composite outcome of a sustained decline in the estimated GFR of at least $50 \%$, end-stage kidney disease, or death from renal or cardiovascular causes than participants who were assigned to receive placebo. Each of the components of the composite outcome occurred less frequently in the dapagliflozin group, with results that were similar for the occurrence of death from cardiovascular causes or hospitalization for heart failure and death from any cause.
Our trial adds to the literature by examining the effect of an SGLT2 inhibitor, added to background therapy including an ACE inhibitor or ARB, in participants with chronic kidney disease, with or without type 2 diabetes.

The kidney-protective effects of SGLT2 inhibitors have previously been shown in patients with type 2 diabetes and chronic kidney disease in the CREDENCE trial. ${ }^{10}$ In that trial, as in most previous cardiovascular outcome trials of SGLT2 inhibitors, the lower estimated GFR cutoff for inclusion was $30 \mathrm{ml}$ per minute per $1.73 \mathrm{~m}^{2}{ }^{16} \mathrm{In}$ contrast to the CREDENCE trial, the present trial examined the effects of an SGLT2 inhibitor in patients with chronic kidney disease of whom $32.5 \%$ did not have type 2 diabetes and $14.5 \%$ had an estimated GFR below $30 \mathrm{ml}$ per minute per $1.73 \mathrm{~m}^{2}$. Our trial confirms that the kidneyprotective effects of SGLT2 inhibitors extend to the broader population of persons with chronic kidney disease without type 2 diabetes, for whom ACE inhibitors are the only pharmacologic treatments that have been shown to prevent kidney failure. $^{3,4}$

Life expectancy is markedly reduced when kidney function declines or albuminuria develops. Global estimates indicate that 1.2 million deaths were attributable to chronic kidney dis- 
ease in 2017. ${ }^{17}$ The findings from the present trial confirm the high mortality among patients with impaired kidney function. The markedly lower mortality in the dapagliflozin group than in the placebo group supports the use of dapagliflozin as an addition to the therapeutic management of chronic kidney disease.

Participants in our trial also had a high risk of hospitalization for heart failure or death from cardiovascular causes. The lower risk of hospitalization for heart failure or death from cardiovascular causes in the dapagliflozin group than in the placebo group is consistent with the results of two previous trials of dapagliflozin, the DECLARE-TIMI 58 (Dapagliflozin Effect on Cardiovascular Events-Thrombolysis in Myocardial Infarction 58) and DAPA-HF (Dapagliflozin and Prevention of Adverse Outcomes in Heart Failure) trials. ${ }^{9}, 18$ Clinical trials of other SGLT2 inhibitors have shown similar results. ${ }^{8,10,19}$

Dapagliflozin had an acceptable safety profile in this population, which included participants with an estimated GFR as low as $25 \mathrm{ml}$ per minute per $1.73 \mathrm{~m}^{2}$. Overall, the present trial confirmed the beneficial safety profile of dapagliflozin. In particular, there were no cases of diabetic ketoacidosis with dapagliflozin, and hypoglycemic episodes did not occur in participants without diabetes.

Our trial has some limitations. First, the trial was stopped on the basis of a recommendation from the independent data monitoring committee. This may have reduced the power of some secondary outcomes. However, the strong inter- nal and external validity of the treatment effect suggests that this limitation is unlikely to have had a major influence on the findings. As in other trials of SGLT2 inhibitors, there was an initial dip in the estimated GFR, followed by a stabilization of kidney-function decline. ${ }^{7,8,10}$ This dip in the estimated GFR reflects favorable hemodynamic changes in the glomerulus. We did not collect estimated GFR values after the completion of the trial and are unable to ascertain whether the initial dip in the estimated GFR is reversible after the discontinuation of dapagliflozin, as observed in other dapagliflozin studies. ${ }^{20}$

The present trial showed that persons with chronic kidney disease who received dapagliflozin had a significantly lower risk of a composite of a sustained decline in the estimated GFR of at least $50 \%$, end-stage kidney disease, or death from renal or cardiovascular causes than those who received placebo, independent of the presence or absence of type 2 diabetes. In addition, those who received dapagliflozin had a lower risk of death from cardiovascular causes or hospitalization for heart failure and had longer survival.

\section{Supported by AstraZeneca.}

Disclosure forms provided by the authors are available with the full text of this article at NEJM.org.

A data sharing statement provided by the authors is available with the full text of this article at NEJM.org.

We thank all the investigators, trial teams, and patients for their participation in the trial; Niels Jongs and Douwe Postmus of the University Medical Center Groningen for independent data verification; and Parita Sheth and Nicola Truss of inScience Communications for assistance in editing and the preparation of figures in an earlier version of the manuscript, funded by AstraZeneca.

APPENDIX

The authors' affiliations are as follows: the Department Clinical Pharmacy and Pharmacology, University of Groningen, University Medical Center Groningen, Groningen, the Netherlands (H.J.L.H.); the George Institute for Global Health, Sydney (H.J.L.H., D.C.W.); Late-Stage Development, Cardiovascular, Renal, and Metabolism, BioPharmaceuticals R\&D, AstraZeneca, Gothenburg, Sweden (B.V.S., M.L., C.D.S., A.-M.L.); the National Medical Science and Nutrition Institute Salvador Zubirán, Mexico City (R.C.-R.); the Departments of Medicine and Epidemiology and Population Health, Stanford University School of Medicine, Stanford, CA (G.M.C.); the Study Design and Biostatistics Center, University of Utah Health Sciences, Salt Lake City (T.G.); the Division of Nephrology, Nanfang Hospital, Southern Medical University, National Clinical Research Center for Kidney Disease, Guangzhou, China (F.-F.H.); KfH Kidney Center, Munich, and Department of Medicine 4, University of Erlangen-Nuremberg, Erlangen — both in Germany (J.F.E.M.); the Institute of Cardiovascular and Medical Sciences, University of Glasgow, Glasgow (J.J.V.M.), and the Department of Renal Medicine, University College London, London (D.C.W.) - both in the United Kingdom; Steno Diabetes Center Copenhagen, Gentofte, and the Department of Clinical Medicine, University of Copenhagen, Copenhagen — both in Denmark (P.R.); and the Department of Internal Medicine, UT Southwestern Medical Center, Dallas (R.D.T.).

REFERENCES

1. Webster AC, Nagler EV, Morton RL, Masson P. Chronic kidney disease. Lancet 2017;389:1238-52.

2. Chatzimanouil MKT, Wilkens L, An- ders H-J. Quantity and reporting quality of kidney research. J Am Soc Nephrol 2019;30:13-22.

3. Ruggenenti P, Perna A, Gherardi G, et al. Renoprotective properties of ACE-inhibition in non-diabetic nephropathies with non-nephrotic proteinuria. Lancet 1999; 354:359-64. 
4. Hou FF, Zhang $X$, Zhang GH, et al. Efficacy and safety of benazepril for advanced chronic renal insufficiency. $\mathrm{N}$ Engl J Med 2006;354:131-40.

5. Brenner BM, Cooper ME, de Zeeuw D, et al. Effects of losartan on renal and cardiovascular outcomes in patients with type 2 diabetes and nephropathy. $\mathrm{N}$ Engl J Med 2001;345:861-9.

6. Lewis EJ, Hunsicker LG, Clarke WR, et al. Renoprotective effect of the angiotensin-receptor antagonist irbesartan in patients with nephropathy due to type 2 diabetes. N Engl J Med 2001;345:851-60.

7. Wanner C, Inzucchi SE, Lachin JM, et al. Empagliflozin and progression of kidney disease in type 2 diabetes. $\mathrm{N}$ Engl $\mathrm{J}$ Med 2016;375:323-34.

8. Neal B, Perkovic V, Mahaffey KW, et al. Canagliflozin and cardiovascular and renal events in type 2 diabetes. $\mathrm{N}$ Engl J Med 2017;377:644-57.

9. Wiviott SD, Raz I, Bonaca MP, et al. Dapagliflozin and cardiovascular outcomes in type 2 diabetes. N Engl J Med 2019;380:347-57.

10. Perkovic V, Jardine MJ, Neal B, et al. Canagliflozin and renal outcomes in type 2 diabetes and nephropathy. $\mathrm{N}$ Engl J Med 2019;380:2295-306.

11. Cherney DZI, Dekkers CCJ, Barbour
SJ, et al. Effects of the SGLT2 inhibitor dapagliflozin on proteinuria in non-diabetic patients with chronic kidney disease (DIAMOND): a randomised, double-blind, crossover trial. Lancet Diabetes Endocrinol 2020;8:582-93.

12. Heerspink HJL, Kosiborod M, Inzucchi SE, Cherney DZI. Renoprotective effects of sodium-glucose cotransporter-2 inhibitors. Kidney Int 2018;94:26-39.

13. van Bommel EJM, Muskiet MHA, van Baar MJB, et al. The renal hemodynamic effects of the SGLT2 inhibitor dapagliflozin are caused by post-glomerular vasodilatation rather than pre-glomerular vasoconstriction in metformin-treated patients with type 2 diabetes in the randomized, double-blind RED trial. Kidney Int 2020;97:202-12.

14. Heerspink HJL, Stefansson BV, Chertow GM, et al. Rationale and protocol of the Dapagliflozin and Prevention of Adverse Outcomes in Chronic Kidney Disease (DAPA-CKD) randomized controlled trial. Nephrol Dial Transplant 2020;35:274-82.

15. Wheeler DC, Stefansson BV, Batiushin $M$, et al. The Dapagliflozin and Prevention of Adverse Outcomes in Chronic Kidney Disease (DAPA-CKD) trial: baseline characteristics. Nephrol Dial Transplant 2020 August 30 (Epub ahead of print).
16. Neuen BL, Young T, Heerspink HJL, et al. SGLT2 inhibitors for the prevention of kidney failure in patients with type 2 diabetes: a systematic review and meta-analysis. Lancet Diabetes Endocrinol 2019;7: 845-54.

17. GBD Chronic Kidney Disease Collaboration. Global, regional, and national burden of chronic kidney disease, 19902017: a systematic analysis for the Global Burden of Disease Study 2017. Lancet 2020; 395:709-33.

18. McMurray JJV, Solomon SD, Inzucchi SE, et al. Dapagliflozin in patients with heart failure and reduced ejection fraction. N Engl J Med 2019;381:1995-2008.

19. Zinman B, Wanner C, Lachin JM, et al. Empagliflozin, cardiovascular outcomes, and mortality in type 2 diabetes. $\mathrm{N}$ Engl J Med 2015;373:2117-28.

20. Pollock C, Stefánsson B, Reyner D, et al. Albuminuria-lowering effect of dapagliflozin alone and in combination with saxagliptin and effect of dapagliflozin and saxagliptin on glycaemic control in patients with type 2 diabetes and chronic kidney disease (DELIGHT): a randomised, double-blind, placebo-controlled trial. Lancet Diabetes Endocrinol 2019;7:429-41.

Copyright @ 2020 Massachusetts Medical Society. 